\title{
Relativistic Langevin Dynamics in Expanding Media
}

\author{
Min He, ${ }^{1,2}$ Hendrik van Hees, ${ }^{3}$ Pol B. Gossiaux, ${ }^{4}$ Rainer J. Fries, ${ }^{1}$ and Ralf Rapp ${ }^{1}$ \\ ${ }^{1}$ Cyclotron Institute and Department of Physics 85 Astronomy, \\ Texas AEM University, College Station, TX 77843, USA \\ ${ }^{2}$ Department of Applied Physics, Nanjing University of Science and Technology, Nanjing 210094, China \\ ${ }^{3}$ Frankfurt Institute for Advanced Studies, Ruth-Moufang-Straße 1, D-60438 Frankfurt, Germany \\ ${ }^{4}$ SUBATECH, UMR 6457, Laboratoire de Physique Subatomique et des Technologies Associées, \\ University of Nantes - IN2P3/CNRS - Ecole des Mines de Nantes, \\ 4 rue Alfred Kastler, F-44072 Nantes Cedex 03, France
}

(Dated: May 28, 2013)

\begin{abstract}
We study the consequences of different realizations of diffusion processes in relativistic Langevin simulations. We elaborate on the Ito-Stratonovich dilemma by showing how microscopically calculated transport coefficients as obtained from a Boltzmann/Fokker-Planck equation can be implemented to lead to an unambiguous realization of the Langevin process. Pertinent examples within the pre-point (Ito) and post-point (Hänggi-Klimontovich) Langevin prescriptions are worked out explicitly. Deviations from this implementation are shown to generate variants of the Boltzmann distribution as the stationary (equilibrium) solutions. Finally, we explicitly verify how the Lorentz invariance of the Langevin process is maintained in the presence of an expanding medium, including the case of an "elliptic flow" transmitted to a Brownian test particle. This is particularly relevant for using heavy-flavor diffusion as a quantitative tool to diagnose transport properties of QCD matter as created in ultrarelativistic heavy-ion collisions.
\end{abstract}

PACS numbers: 12.38.Mh, 05.10.Gg, 25.75.-q

Keywords: Brownian Motion, Relativistic Langevin simulation, Boltzmann-Jüttner distribution, Heavyquark diffusion

\section{INTRODUCTION}

Since its introduction more than 100 years ago, Brownian motion has remained a valuable and versatile tool to study a wide variety of physical systems. In recent years considerable efforts have been devoted to its extension and reliable implementation for relativistic systems [15]. In particular, it has been applied in the studies of heavy-quark (HQ) diffusion in the Quark-Gluon Plasma (QGP) 6 - 10], in order to evaluate transport properties of the medium produced in ultrarelativistic heavy-ion collisions (URHICs) (see, e.g., Ref. [11] for a recent review). Since charm- and bottom-quark masses are much larger than the typical temperatures, as well as the constituent masses of the equilibrated medium, a separation of the HQ relaxation time and collision time emerges [8, 11], thus justifying a soft-collision approximation to be accommodated by Fokker-Planck dynamics [12]. In practice, the Fokker-Planck equation is routinely realized by stochastic Langevin processes [2, 3, 11, 13]. However, it is known that the implementation of the Langevin equation is not unique but depends on the realization of the stochastic integral, resulting in (seemingly) different Fokker-Planck equations, known as the "Ito-Stratonovich dilemma" [2, 3, 13]. Moreover, the ambiguities in the Langevin discretization scheme raise concerns on the asymptotic phase-space distribution of the relativistic Brownian particles under consideration. The latter has been an issue of debate (see Ref. 14] and references therein) but should be constrained by the long-time limit of equilibrium [3, 12, 15, 22], i.e., by a "detailedbalancing" of the drag and diffusion terms of the under- lying Fokker-Planck equation.

The large collective flow of the medium created in URHICs (reaching collective velocities in excess of half the speed of light), and in particular subtle angular modulations thereof (such as the elliptic flow), make a good understanding of differences in the realization of relativistic Langevin simulations mandatory. In particular, the description of the phase-space distribution of a Brownian particle (the heavy quark), which is usually formulated in laboratory-frame coordinates, needs to manifestly recover the equilibrium limit given by the Lorentz-invariant Boltzmann-Jüttner distribution [18, 19]. This is evident as the underlying Fokker-Planck equation is to yield a good approximation of the Lorentz-covariant Boltzmann equation [1, 12, 13. This problem has been addressed, e.g., in Refs. 15, 23] within the so-called Ito realization, while pertinent Fokker-Planck equations following from different Langevin prescriptions have been given in Ref. 3]. More recently, a Lorentz-covariant implementation of the Langevin process has been discussed in Ref. 24] based on a relativistic fluctuation-dissipation theorem.

In the present work we pursue a slightly different approach. We start from a Fokker-Planck equation with transport coefficients obtained from an underlying microscopic theory, in the sense of an approximate treatment of the collision integral in the transport equation. After recovering the generalized relativistic Einstein relation for these coefficients, we discuss their implementation into different Langevin realizations and verify their uniqueness in explicit examples, in particular including the case of expanding media. We also show how vari- 
ations in the Langevin coefficients induce modifications to the long-time limit (equilibrium) distribution of the Brownian particle. For definiteness, our numerical applications will be illustrated in the context of HQ diffusion in an expanding QGP as formed in URHICs.

Our article is organized as follows. In Sec. II we introduce the Fokker-Planck equation as an approximate description of the Boltzmann equation, and recall the general equilibrium condition constraining its drag and diffusion coefficients. In Sec. III we elaborate how Langevin prescriptions emerge from the pertinent equilibrium conditions to ensure a uniform outcome of the Fokker-Planck framework, i.e., how Fokker-Planck equations resulting from different Langevin prescriptions take the same form in terms of universal drag and diffusion coefficients of an underlying microscopic theory. In Sec. IV]Langevin prescriptions are illustrated with numerical calculations for different model systems for flowing media. We demonstrate that both pre- and post-point Langevin schemes lead to the Lorentz-invariant Boltzmann-Jüttner distribution for test particles in the presence of collective flow as the long-time limit of the Langevin process, if and only if the proper equilibrium condition is imposed. Some variants of the realization of the stochastic Langevin process and their consequences on the long-time limit are also discussed in this section. We summarize and conclude in Sec. V

\section{FOKKER-PLANCK EQUATION AND GENERAL EQUILIBRIUM CONDITION}

When a heavy particle is immersed into a medium of light constituents at small to moderate temperature, $T \lesssim m$, its momentum change due to collisions is relatively small. Employing this soft-scattering approximation [12], the Boltzmann integro-differential equation describing the motion of the heavy particles in an equilibrated background medium reduces to the Fokker-Planck equation for the phase-space distribution function, $f$, of the "Brownian" particle 12]

$$
\frac{\partial f(t, \boldsymbol{p})}{\partial t}=\frac{\partial}{\partial p_{i}}\left\{A_{i}(\boldsymbol{p}) f(t, \boldsymbol{p})+\frac{\partial}{\partial p_{j}}\left[B_{i j}(\boldsymbol{p}) f(t, \boldsymbol{p})\right]\right\}
$$

with $i, j \in\{1,2,3\}$. We focus on a spatially homogeneous static medium without external force, and thus the phase-space density is independent of $\boldsymbol{x}$. The drag and diffusion coefficients in Eq. (11), $A_{i}(\boldsymbol{p})$ and $B_{i j}(\boldsymbol{p})$, respectively, are obtained from an average of the moments of momentum transfer in heavy-light collisions, weighted by a transition probability given by the pertinent scattering matrix elements, $\mathcal{M}$, and the medium particle distribu- tion, $f^{p}$,

$$
\begin{aligned}
A_{i}(\boldsymbol{p})= & \frac{1}{2 E(\boldsymbol{p})} \int \frac{\mathrm{d}^{3} \boldsymbol{q}}{(2 \pi)^{3} 2 E(\boldsymbol{q})} \int \frac{\mathrm{d}^{3} \boldsymbol{q}^{\prime}}{(2 \pi)^{3} 2 E\left(\boldsymbol{q}^{\prime}\right)} \\
& \times \int \frac{\mathrm{d}^{3} \boldsymbol{p}^{\prime}}{(2 \pi)^{3} 2 E\left(\boldsymbol{p}^{\prime}\right)} \frac{1}{\gamma} \sum|\mathcal{M}|^{2} \hat{f}(\boldsymbol{q}) \\
& (2 \pi)^{4} \delta^{4}\left(p+q-p^{\prime}-q^{\prime}\right)\left[\left(\boldsymbol{p}^{\prime}-\boldsymbol{p}\right)_{i}\right] \\
\equiv & \left\langle\left\langle\left(\boldsymbol{p}^{\prime}-\boldsymbol{p}\right)_{i}\right\rangle\right\rangle, \\
B_{i j}(\boldsymbol{p})= & \frac{1}{2}\left\langle\left\langle\left(\boldsymbol{p}^{\prime}-\boldsymbol{p}\right)_{i}\left(\boldsymbol{p}^{\prime}-\boldsymbol{p}\right)_{j}\right\rangle\right\rangle .
\end{aligned}
$$

Here, $\boldsymbol{p}$ and $\boldsymbol{p}^{\prime}$ ( $\boldsymbol{q}$ and $\boldsymbol{q}^{\prime}$ ) are the heavy (light) particle's momentum before and after the collision, respectively, and $\hat{f}=f^{p}\left(1 \pm f^{p}\right)$ for bosons/fermions in the medium.

Defining a current

$$
S_{i}(t, \boldsymbol{p})=-\left\{A_{i}(\boldsymbol{p}) f(t, \boldsymbol{p})+\frac{\partial}{\partial p_{j}}\left[B_{i j}(\boldsymbol{p}) f(t, \boldsymbol{p})\right]\right\},
$$

the Fokker-Planck equation can be cast as a continuity equation in momentum space [12],

$$
\frac{\partial f_{Q}(t, \boldsymbol{p})}{\partial t}+\frac{\partial}{\partial p_{i}} S_{i}(t, \boldsymbol{p})=0 \text {. }
$$

This identifies $S_{i}$ as a particle-number current (flux), so that the number of heavy particles is conserved in the diffusion process. After sufficiently many collisions with the light partons, i.e., in the long-time limit, we expect the heavy particle to approach the same equilibrium distribution as for the medium constituents given by the relativistic Boltzmann-Jüttner distribution [20],

$$
f_{\text {eq }}(p, T)=N \exp [-E(p) / T],
$$

where $E(p)=\sqrt{\boldsymbol{p}^{2}+m^{2}}$ is the relativistic on-shell energy of the Brownian particle and $T$ the temperature of the equilibrated medium. In addition, in statistical equilibrium, the particle flux has to vanish [12]: $S_{i}(\boldsymbol{p})=0$. Together with the Boltzmann-Jüttner distribution (6) this yields a dissipation-fluctuation relation between drag and diffusion coefficient,

$$
A_{i}(\boldsymbol{p}, T)=B_{i j}(\boldsymbol{p}, T) \frac{1}{T} \frac{\partial E(p)}{\partial p_{j}}-\frac{\partial B_{i j}(\boldsymbol{p}, T)}{\partial p_{j}} .
$$

This is the manifestation of the detailed-balance property of the collision-transition probabilities, which is due to the unitarity of the $S$-matrix of quantum-field theory and thus embodied in the collision integral of the underlying full Boltzmann equation [12]. This relation is not a priori fulfilled by Eqs. (2) and (3), and may suffer in accuracy if the assumption of a forward-peaked transition-matrix element is not well satisfied. In order for the heavy particle to approach the same distribution as for the medium particles, the drag and diffusion coefficients are not independent but have to be related to each other precisely as specified by the dissipation-fluctuation relation (7). This general equilibrium condition plays a central role in the following discussions. 


\section{STOCHASTIC LANGEVIN REALIZATION OF FOKKER-PLANCK DIFFUSION}

\section{A. Langevin Simulation}

The Fokker-Planck description of diffusion can be realized by Langevin's stochastic differential equation(s) [3, 11, 13. Employing a spatially homogeneous static medium, we follow the notation in Ref. [11] to write the Langevin equations as

$$
\begin{aligned}
\mathrm{d} x_{j} & =\frac{p_{j}}{E} \mathrm{~d} t, \\
\mathrm{~d} p_{j} & =-\Gamma(p, T) p_{j} \mathrm{~d} t+\sqrt{\mathrm{d} t} C_{j k}(\boldsymbol{p}+\xi d \boldsymbol{p}, T) \rho_{k},
\end{aligned}
$$

which specify the rules for updating the coordinate and momentum of the heavy particle in time steps, $\mathrm{d} t$. In Eq. (9), $\Gamma(p, T) \boldsymbol{p}$ is the deterministic friction force, whereas the $C_{j k}$ describe the stochastically fluctuating force with independent Gaussian noises $\rho_{k}$ following a normal distribution, $P(\boldsymbol{\rho})=(2 \pi)^{-3 / 2} e^{-\boldsymbol{\rho}^{2} / 2}$. Thus, there is no correlation in the stochastic forces at different times, $\left\langle F_{j}(t) F_{k}\left(t^{\prime}\right)\right\rangle=C_{j l} C_{k l} \delta\left(t-t^{\prime}\right)$, consistent with the assumption of uncorrelated momentum kicks underlying the Fokker-Planck equation ("white noise"). However, it is not specified at what momentum argument the covariance matrix should be evaluated: $C_{j k}=C_{j k}(t, \boldsymbol{p}+\xi \mathrm{d} \boldsymbol{p})$, with $\xi \in[0,1]$, e.g., $\xi=0$ for pre-point (Ito) [21], $\xi=1 / 2$ for mid-point (Stratonovich), or $\xi=1$ for post-point (Hänggi-Klimontovich) [2] realizations of the stochastic integral. We will return to this point below.

The phase-space distribution determined by the Langevin equations (8) and (9) satisfies a Fokker-Planck equation, which can be found by calculating the average change of an arbitrary phase-space function with time [11]. The resulting equation reads

$$
\begin{array}{r}
\frac{\partial f(t, \boldsymbol{p})}{\partial t}=\frac{\partial}{\partial p_{j}}\left[\left(\Gamma(p) p_{j}-\xi C_{l k}(\boldsymbol{p}) \frac{\partial C_{j k}(\boldsymbol{p})}{\partial p_{l}}\right) f(t, \boldsymbol{p})\right] \\
+\frac{1}{2} \frac{\partial^{2}}{\partial p_{j} \partial p_{k}}\left[C_{j l}(\boldsymbol{p}) C_{k l}(\boldsymbol{p}) f(t, \boldsymbol{p})\right] .
\end{array}
$$

Comparing Eqs. (10) and (10) leads to

$$
\begin{aligned}
A_{j}(\boldsymbol{p}) & =A(p) p_{j}=\Gamma(p) p_{j}-\xi C_{l k}(\boldsymbol{p}) \frac{\partial C_{j k}(\boldsymbol{p})}{\partial p_{l}}, \\
B_{j k}(\boldsymbol{p}) & =B_{0}(p) P_{j k}^{\perp}(\boldsymbol{p})+B_{1}(p) P_{j k}^{\|}(\boldsymbol{p}) \\
& =\frac{1}{2} C_{j l}(\boldsymbol{p}) C_{k l}(\boldsymbol{p})
\end{aligned}
$$

where $P_{j k}^{\perp}(\boldsymbol{p})=\delta_{j k}-p_{j} p_{k} / p^{2}$ and $P_{j k}^{\|}(\boldsymbol{p})=p_{j} p_{k} / p^{2}$ are the corresponding projection operators, so that

$$
C_{j k}(\boldsymbol{p})=\sqrt{2 B_{0}(p)} P_{j k}^{\perp}(\boldsymbol{p})+\sqrt{2 B_{1}(p)} P_{j k}^{\|}(\boldsymbol{p}) .
$$

Two comments are in order here. First, note that the friction coefficient, $\Gamma(p, T)$, appearing in the
Langevin equation (9), is a derived quantity, given in terms of the drag and diffusion coefficients, $A(p, T)$, $B_{0}(p, T)$ and $B_{1}(p, T)$, as determined by microscopic scattering-transition matrix elements (e.g., they relate to heavy-quark energy loss due to elastic heavy-light collisions 26] ). Second, the Fokker-Planck equation and the Langevin equation are specified for a spatially homogeneous static medium; neither of them is manifestly Lorentz covariant. Thus, in the case of a flowing medium, momentum updates in the Langevin equation (9) have to be calculated in the local fluid-rest frame and then boosted back to the moving frame when performing the diffusion simulations. It is readily proved that the coordinate updates (8) can be equivalently done in the moving frame; hereafter, we refer to the latter as the laboratory frame.

\section{B. Evaluation of Equilibrium Condition}

We now proceed to analyze the equilibrium condition for two implementations of the Langevin process, the prepoint and post-point prescriptions, and work out their manifestations in the Fokker-Planck framework. For the sake of brevity, but without loss of generality, we work with a diagonal approximation of the diffusion coefficient from here on. Putting $B_{0}(p)=B_{1}(p)=D(p)$, the covariance matrix (13) reduces to $C_{j k}(\boldsymbol{p})=\sqrt{2 D(p)} \delta_{j k}$. Utilizing Eq. (11), the general equilibrium condition (7) simplifies to

$$
A(p)=\frac{1}{E(p)}\left(\frac{D[E(p)]}{T}-\frac{\partial D[E(p)]}{\partial E}\right)
$$

with

$$
\Gamma(p)=\frac{1}{E(p)}\left(\frac{D[E(p)]}{T}-(1-\xi) \frac{\partial D[E(p)]}{\partial E}\right) .
$$

Thus, for a given drag coefficient $A$, Eq. (14) should be solved to obtain the diffusion coefficient $D$, which can then be used to deduce the friction force $\Gamma$ from Eq. (15). Alternative procedures have been adopted in the literature by, e.g., calculating $D$ and adjusting $A[8,25]$. Condition (15) has also been derived as the generalization of the fluctuation-dissipation theorem with BoltzmannJüttner distributions as the equilibrium solution in 24].

In the non-relativistic limit, both $D(p)=D$ and $\Gamma(p)=\gamma$ become independent of $p$, and $E(p) \rightarrow m$; the equilibrium condition reduces to

$$
D=m \gamma T,
$$

which is Einstein's classical fluctuation-dissipation relation 12 .

In terms of the diagonal diffusion coefficient, the Langevin updating rules now read

$$
\begin{aligned}
\mathrm{d} x_{j} & =\frac{p_{j}}{E} \mathrm{~d} t, \\
\mathrm{~d} p_{j} & =-\Gamma(p) p_{j} \mathrm{~d} t+\sqrt{2 \mathrm{~d} t D(|\boldsymbol{p}+\xi d \boldsymbol{p}|)} \rho_{j} .
\end{aligned}
$$


In the following, we discuss their explicit forms in the prepoint and post-point Langevin prescriptions as following from the corresponding equilibrium condition, and show that they can both be rendered compatible for a given microscopic model.

\section{Pre-point scheme: $\xi=0$}

In this scheme, the equilibrium conditions (14) and (15) read

$$
\Gamma(p)=\frac{1}{E(p)}\left(\frac{D[E(p)]}{T}-\frac{\partial D[E(p)]}{\partial E}\right)=A(p) .
$$

Note that the friction force entering the Langevin equation is equal to the drag coefficient defined by Eq. (2).

Inserting $C_{j k}(\boldsymbol{p})=\sqrt{2 D(p)} \delta_{j k}$ into Eq. (10), the Fokker-Planck equation realized in the pre-point Langevin scheme takes the form

$$
\frac{\partial}{\partial t} f(t, \boldsymbol{p})=\frac{\partial}{\partial p_{i}}\left\{\Gamma(p) p_{i} f(t, \boldsymbol{p})+\frac{\partial}{\partial p_{i}}[D(p) f(t, \boldsymbol{p})]\right\} .
$$

The corresponding Langevin updating rules now read

$$
\begin{aligned}
\mathrm{d} x_{j} & =\frac{p_{j}}{E} \mathrm{~d} t, \\
\mathrm{~d} p_{j} & =-\Gamma(p) p_{j} \mathrm{~d} t+\sqrt{2 \mathrm{~d} t D(p)} \rho_{j},
\end{aligned}
$$

where carrying out the Langevin time steps is straightforward: starting with initial coordinate $\boldsymbol{x}$ and momentum $\boldsymbol{p}$ of a test particle, calculate the coordinate increment $\mathrm{d} x_{j}$ within the time step $\mathrm{d} t$ using Eq. (21); at the same time substitute the drag coefficient $A(p)(=\Gamma(p))$ evaluated at $p=|\boldsymbol{p}|$ and the corresponding $D(p)$ calculated from Eq. (14) into Eq. (22) to calculate the momentum increment. Then go to the next time-step with (new) initial coordinate $\boldsymbol{x}+\mathrm{d} \boldsymbol{x}$ and momentum $\boldsymbol{p}+\mathrm{d} \boldsymbol{p}$.

\section{Post-point scheme: $\xi=1$}

In this scheme, the equilibrium conditions read

$$
\begin{aligned}
D[E(p)] & =\Gamma(p) E(p) T \\
\Gamma(p) & =A(p)+\frac{1}{E(p)} \frac{\partial D[E(p)]}{\partial E} .
\end{aligned}
$$

Here, the scheme-dependent relation takes a simple form, but the "price" to pay is that the friction force $\Gamma(p)$ figuring into the Langevin equation is different from the drag coefficient $A(p)$. In this sense, starting from a microscopic model for $A(p)$, there is no difference in the choice of the pre-point or the post-point scheme.

The corresponding Fokker-Planck equation in the postpoint scheme takes the form

$$
\frac{\partial}{\partial t} f(t, \boldsymbol{p})=\frac{\partial}{\partial p_{i}}\left\{\Gamma(p) p_{i} f(t, \boldsymbol{p})+D(p) \frac{\partial}{\partial p_{i}} f(t, \boldsymbol{p})\right\} .
$$

This Fokker-Planck equation appears to be different from its counterpart realized in the pre-point realization, Eq. (20). However, upon substituting the different expressions for $\Gamma$, Eqs. (19) and (24), for the prepoint and the post-post Langevin scheme into Eqs. 20. and (25), respectively, one immediately sees that the Fokker-Planck equations realized in the pre- and postpoint Langevin schemes are actually the same in terms of $A(p)$ and $D(p)$. This is a desired result since the (approximate) realization of the underlying full Boltzmann equation (including the asymptotic solution for the distribution of test particles) within a Fokker-Planck equation should be independent of the implementation of the stochastic process (i.e., Langevin scheme). Thus, the "Ito-Stratonovich dilemma", which suggests that different Langevin prescriptions are not sufficient in order to uniquely determine the Fokker-Planck equation, does not appear here, as a benefit of a well-defined microscopic process.

The Langevin updating rules in the post-point scheme read

$$
\begin{aligned}
\mathrm{d} x_{j} & =\frac{p_{j}}{E} \mathrm{~d} t \\
\mathrm{~d} p_{j} & =-\Gamma(p) p_{j} \mathrm{~d} t+\sqrt{2 \mathrm{~d} t D(|\boldsymbol{p}+d \boldsymbol{p}|)} \rho_{j} .
\end{aligned}
$$

Starting with initial coordinate $\boldsymbol{x}$ and momentum $\boldsymbol{p}$, the coordinate update is trivial at given time step $\mathrm{d} t$, but the momentum update involves a two-step computation: first use the pre-point scheme Eq. (22) to calculate the momentum increment $\mathrm{d} \boldsymbol{p}$ with $\Gamma(p)$ and $D(p)$; then evaluate the diffusion coefficient $D$ at the argument $|\boldsymbol{p}+\mathrm{d} \boldsymbol{p}|$ and calculate $\mathrm{d} p_{j}^{\text {diffusion }} \equiv \sqrt{2 \mathrm{~d} t D(|\boldsymbol{p}+d \boldsymbol{p}|)} \rho_{j}$, while $\mathrm{d} p_{j}^{\mathrm{drag}}=-\Gamma(p) p_{j} \mathrm{~d} t$ has already been calculated

in the first step; finally, add $\mathrm{up} \mathrm{d} p_{j}^{\text {diffusion }}$ and $\mathrm{d} p_{j}^{\mathrm{drag}}$ to obtain the total momentum increment for the present time-step. Note that in the two-step momentum update procedure, the same $\mathrm{d} t$ and $\rho_{j}$ should be used. A variant where different $\mathrm{d} t$ 's are used will be discussed in the case of a flowing medium in Secs. IVB and IV C below.

\section{NUMERICAL CALCULATIONS WITH DIFFERENT BACKGROUND MEDIA}

In this section we perform numerical simulations of the two different Langevin prescriptions discussed above (and some variants thereof previously employed in the literature), to explicitly examine their consequences for the long-time limits, in particular in the case of flowing media. For definiteness we will adopt parameter values for masses, temperature and flow profiles representing the problem of heavy-quark diffusion in a QGP. We will start with a homogeneous static medium (Sec. IV A), followed by a simple one-dimensional flow scenario (Sec. IVB ) and finally study a more realistic elliptically expanding fireball (Sec. IVC). In all cases we use large drag and diffusion coefficients and perform the simulations until the 

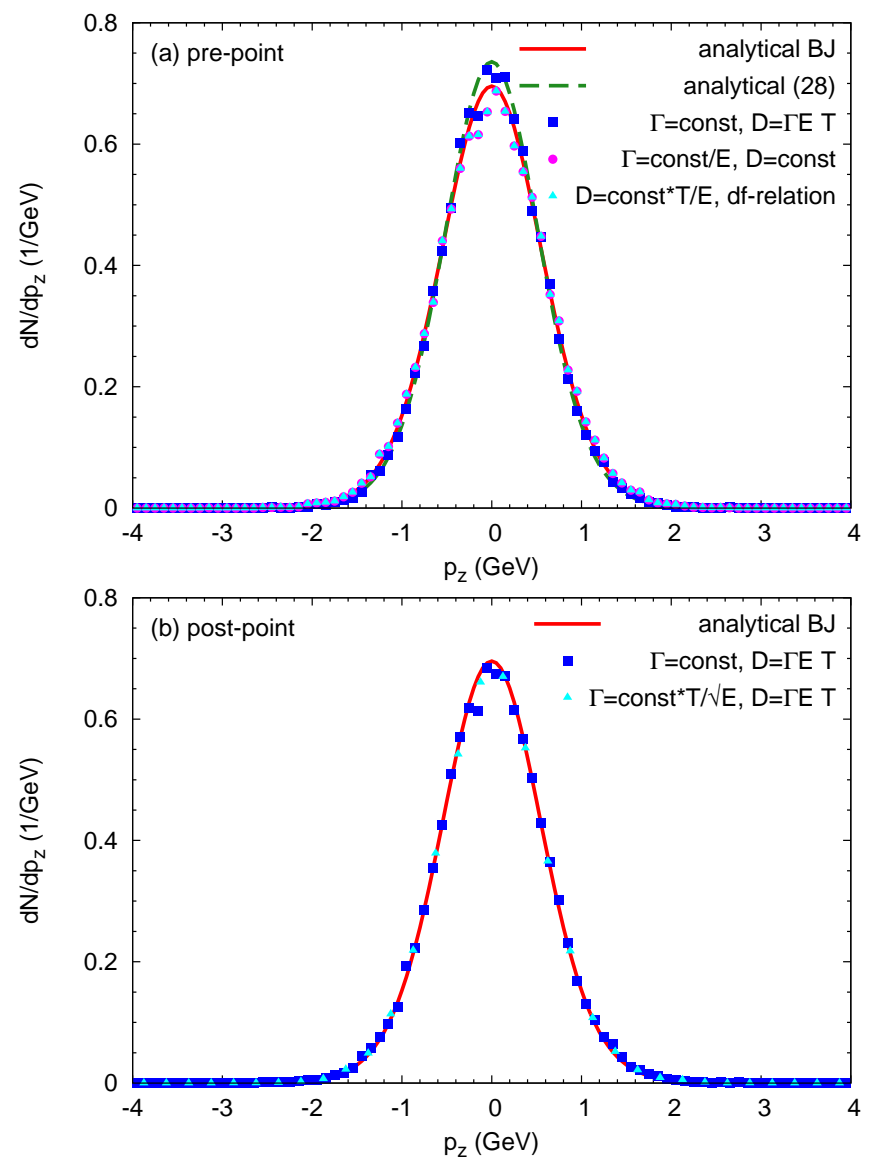

FIG. 1: The distribution, $\mathrm{d} N / \mathrm{d} p_{z}$, from Langevin simulations for heavy quarks with mass $m=1.5 \mathrm{GeV}$, diffusing in a static medium at temperature $T=0.18 \mathrm{GeV}$, compared to calculations with the corresponding analytical phase-space distributions: (a) pre-point Langevin scheme, (b) post-point Langevin scheme. See the text for more details.

stationary state has been reached, demonstrating their universal convergence to the analytical solutions for the corresponding Fokker-Planck equations.

\section{A. Homogeneous Static Medium}

Let us assume a homogeneous static medium with temperature $T=0.18 \mathrm{GeV}$ with a Brownian particle of mass $m=1.5 \mathrm{GeV}$ immersed (we work in natural units where the velocity of light, Planck constant, and Boltzmann constant are equal to unity: $c=\hbar=k_{\mathrm{B}}=1$ ). The preceding Fokker-Planck and Langevin equations can be readily applied; we focus on the projection on the $z$ component of the momentum distribution, $\mathrm{d} N / \mathrm{d} p_{z}$ of the test particle. It has been checked that the equilibrium conditions are reached independent of arbitrarily chosen initial conditions, as expected.

We first adopt the pre-point scenario in a simplistic scenario where a large constant friction coefficient $(\Gamma=$ $20 \mathrm{fm}^{-1}$ ) is complemented by a diffusion coefficient $D=$
$\Gamma E T$. This leads to a stationary distribution,

$$
\mathrm{d} N / \mathrm{d}^{3} x \mathrm{~d}^{3} p=m / E \exp [-E(p) / T],
$$

as shown in the upper panel of Fig. 1. It can be easily verified analytically by plugging the drag and diffusion coefficients into the Fokker-Planck equation (20) and performing the momentum derivatives, as was also discussed in Ref. 2]. However, this form of drag and diffusion coefficient does not comply with the pre-point equilibrium condition (19), and thus the simulation fails to converge to the Boltzmann-Jüttner distribution.

To illustrate the importance of implementing the proper equilibrium condition, we have performed Langevin simulations within the pre-point scheme using two types of momentum dependencies of the diffusion coefficient:

$$
\text { (i) } \begin{aligned}
& \Gamma=20 / E \mathrm{GeV} / \mathrm{fm} ; \\
& D=\Gamma E T=20 T \mathrm{GeV} / \mathrm{fm} . \\
& \text { (ii) } \Gamma=300(1+T / E) / E^{2} \mathrm{GeV}^{2} / \mathrm{fm} ; \\
& D=300 T / E \mathrm{GeV}^{2} / \mathrm{fm} .
\end{aligned}
$$

The lifetime of the system in these simulations has been set to $10 \mathrm{fm}$, and it has been checked that the stationary distribution has been reached.

The simulated results are also shown in the upper panel of Fig. 1. Again, their agreement with the BoltzmannJüttner distribution can be analytically verified by substituting the coefficients into the Fokker-Planck equation (20) and carrying out the momentum derivatives.

Next, we employ the post-point scenario. The simulated results are shown in the lower panel of Fig. 1. Here, the Langevin simulations with large drag reproduce well the Boltzmann-Jüttner distribution, whether the friction is constant $(\Gamma=20 / \mathrm{fm}, D=\Gamma E T)$ or not $\left(\Gamma=40 \sqrt{\mathrm{GeV}_{\mathrm{fm}}}{ }^{-1} / \sqrt{E(p)}, D=\Gamma E T\right)$, since the postpoint equilibrium condition (23) is always fulfilled. This is easily verified by plugging the coefficients into the corresponding Fokker-Planck equation (25).

To summarize this part, we have confirmed through numerical simulations that in order for the stationary Langevin limit to converge to the Boltzmann-Jüttner distribution, the equilibrium condition must be exactly fulfilled.

\section{B. Constant 1-D Flow}

We now introduce a constant one-dimensional medium flow with velocity field $v_{x}=0, v_{y}=0$ and $v_{z}=0.9$. The medium temperature, $T=0.18 \mathrm{GeV}$, and heavy-quark mass, $m=1.5 \mathrm{GeV}$, are as before.

The numerical simulations in the pre-point scheme directly translate the result for the static medium into the corresponding "blast-wave" distribution, obtained by replacing $E$ with $p \cdot u$. Specifically, a momentumindependent drag complemented by the naive $D=$ 

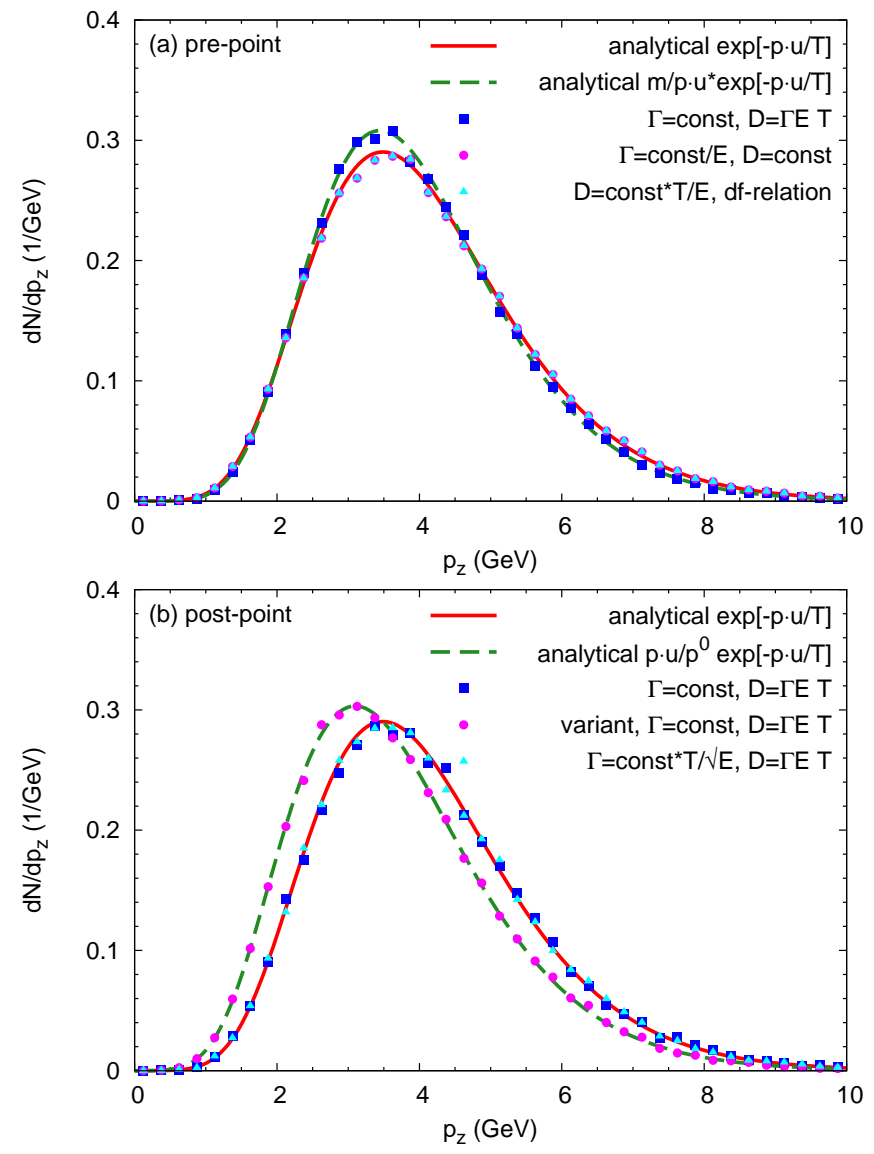

FIG. 2: Langevin simulation results for heavy quarks ( $m=$ $1.5 \mathrm{GeV})$ diffusing in a flowing medium $(T=0.18 \mathrm{GeV}$, $\left.v_{z}=0.9\right)$ compared to calculations with analytical phasespace distributions: (a) pre-point Langevin scheme, (b) postpoint Langevin scheme. The distribution obtained with a variant of post-point scheme and the corresponding blastwave distribution are also shown. See text for more details.

$\Gamma E T$ leads to a single-particle phase-space distribution $\mathrm{d} N / \mathrm{d}^{3} x \mathrm{~d}^{3} p=m /(p \cdot u) \exp (-p \cdot u / T)$, while general momentum-dependent coefficients that fulfill the prepoint equilibrium condition (19) give the BoltzmannJüttner distribution, $\mathrm{d} N / \mathrm{d}^{3} x \mathrm{~d}^{3} p=\exp (-p \cdot u / T)$, see upper panel of Fig. IVB

For the post-point scenario, where the equilibrium condition takes the simple form (23), the momentum update involves two steps in the fluid-rest frame. There seems to be an ambiguity in the time increment $\mathrm{d} t^{*}$ used in the two-step computation (here and in the following, variables with (without) superscript $*$ refer to the fluid rest (laboratory) frame). For the first step, it is clear that $\mathrm{d} t_{(1)}^{*}=\mathrm{d} t \frac{E^{*}}{E}=\mathrm{d} t \frac{p \cdot u}{p^{0}}$ with $E=p^{0}$ being the lab-frame energy prior to the momentum update and $E^{*}=p \cdot u$ the corresponding energy measured in the fluid rest frame. One then updates the momentum and obtains $p_{(1)}^{* \mu}=\left(E_{(1)}^{*}, \boldsymbol{p}_{(1)}^{*}\right)$ in the fluid rest frame; the corresponding lab-frame four-momentum, $p_{(1)}^{\mu}=\left(E_{(1)}, \boldsymbol{p}_{(1)}\right)$, follows from a boost using the fluid
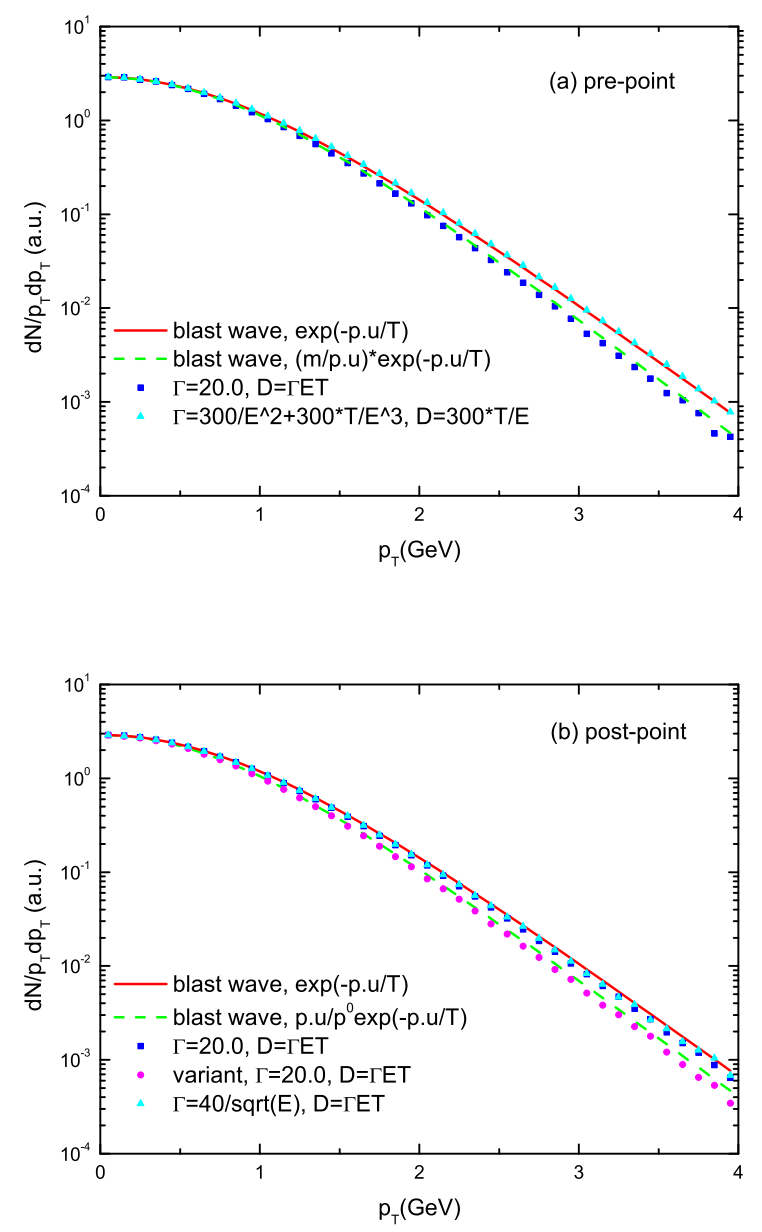

FIG. 3: Langevin simulation of the $p_{T}$-spectrum of a heavy quark $(m=1.5 \mathrm{GeV})$ diffusing in a fireball $\left(T_{i}=0.33 \mathrm{GeV}\right.$, $\left.T_{f}=0.18 \mathrm{GeV}\right)$, compared to the direct blast wave calculations. Upper panel: pre-point Langevin scheme; lower panel: post-point Langevin scheme. See text for more details.

velocity $u^{\mu}$. In the subsequent second-step momentum update, one finds a particle phase-space distribution, $\mathrm{d} N / \mathrm{d}^{3} x \mathrm{~d}^{3} p=\left(p \cdot u / p^{0}\right) \exp (-p \cdot u / T)$ in the lab frame if one uses $\mathrm{d} t_{(2)}^{*}=\mathrm{d} t \frac{E_{(1)}^{*}}{E_{(1)}}=\mathrm{d} t \frac{p_{(1)} \cdot u}{E_{(1)}} \neq \mathrm{d} t_{(1)}^{*}$. This is different from the Boltzmann-Jüttner distribution (we denote it as a "variant"), as illustrated in the lower panel of Fig. IVB One finds both analytically and numerically that the Boltzmann-Jüttner distribution is recovered when using $\mathrm{d} t_{(2)}^{*}=\mathrm{d} t_{(1)}^{*}$ in the second-step momentum update. Recalling that in the general derivation of Fokker-Planck equation from the Langevin equations [11] (for a static thermal medium) a fixed $\mathrm{d} t^{*}=\mathrm{d} t_{(1)}^{*}$ is adopted, we conclude that in the post-point scheme, one should use the same $\mathrm{d} t^{*}=\mathrm{d} t_{(1)}^{*}$ for both momentumupdate steps in order to obtain the Lorentz-invariant Boltzmann-Jüttner distribution in the presence of flow. 


\section{Elliptic Fireball}

In non-central heavy-ion collisions, interactions among particles convert the initial spatial asymmetry into particle momentum anisotropies, most notably an "elliptic flow" quantified by the coefficient of the second harmonic, $v_{2}\left(p_{T}\right)$, in the azimuthal-angle distribution of the particle spectrum [16, 17],

$$
\frac{\mathrm{d} N}{p_{T} \mathrm{~d} p_{T} \mathrm{~d} \phi_{p}}=\frac{\mathrm{d} N}{2 \pi p_{T} \mathrm{~d} p_{T}}\left[1+2 v_{2}\left(p_{T}\right) \cos \left(2 \phi_{p}\right)+\cdots\right]
$$

where $p_{T}$ is the transverse momentum of the emitted particle. Heavy quarks (or hadrons containing heavy quarks) are believed to acquire a $v_{2}$ through the coupling to the collective motion of the light particles in the QGP [8, 11]. In the following, we employ an elliptically expanding fireball, introduced in Ref. [27] (see also the discussion in Ref. [28] for more details) to model the QGP medium QGP evolution, in order to scrutinize the Langevin simulation of heavy-quark (HQ) diffusion in the QGP background. We again take the $\mathrm{HQ}$ mass to be $m=1.5 \mathrm{GeV}$ and employ the same two sets of coefficients $\Gamma$ and $D$ as specified in Eqs. (29) and (30), satisfying the equilibrium conditions. This time the Langevin simulation runs in parallel to an isentropically expanding fireball which stops at the decoupling temperature $T_{f}=0.18 \mathrm{GeV}$. We compute the spectrum and elliptic flow and compare to the results of a direct blast-wave calculation which corresponds to the equilibrium limit of the fireball.

Let us first discuss the HQ $p_{T}$-spectrum, shown in Fig. 3 For the pre-point scenario (upper panel), Langevin simulation results translate again directly into the corresponding blast-wave distributions (obtained by replacing $E$ with $p \cdot u$ ). For a momentum-independent drag coefficient complemented by the naive $D=\Gamma E T$ (at variance with the equilibrium condition), the HQ phase-space distribution assumes the form $\mathrm{d} N / \mathrm{d}^{3} x \mathrm{~d}^{3} p=$ $m /(p \cdot u) \exp (-p \cdot u / T)$, while general momentum dependent coefficients that fulfill the pre-point equilibrium condition (19) accurately recover the Boltzmann-Jüttner distribution $\mathrm{d} N / \mathrm{d}^{3} x \mathrm{~d}^{3} p=\exp (-p \cdot u / T)$. For the postpoint scenario (lower panel), again, once the corresponding equilibrium condition (23) is satisfied, the Langevin simulation also yields the correct Lorentz-invariant Boltzmann distribution, while the "variant" scheme leads to an explicitly frame dependent distribution $\mathrm{d} N / \mathrm{d}^{3} x \mathrm{~d}^{3} p=$ $\left(p \cdot u / p^{0}\right) \exp (-p \cdot u / T)$. This results from using a different $\mathrm{d} t_{(2)}^{*} \neq \mathrm{d} t_{(1)}^{*}$ in the two-step momentum update, as discussed in Sec. IVB.

The HQ $v_{2}\left(p_{T}\right)$ obtained from the equilibrium limit of the Langevin simulations and its comparison with the direct blast-wave calculations are shown in Fig. [4 The agreement between different implementations (prepoint, post-point and variants) of Langevin simulations and the corresponding blast-wave distributions confirm the conclusions for the $p_{T}$ spectra, reproducing accurately even rather subtle angular modulations in relativistic flow fields.
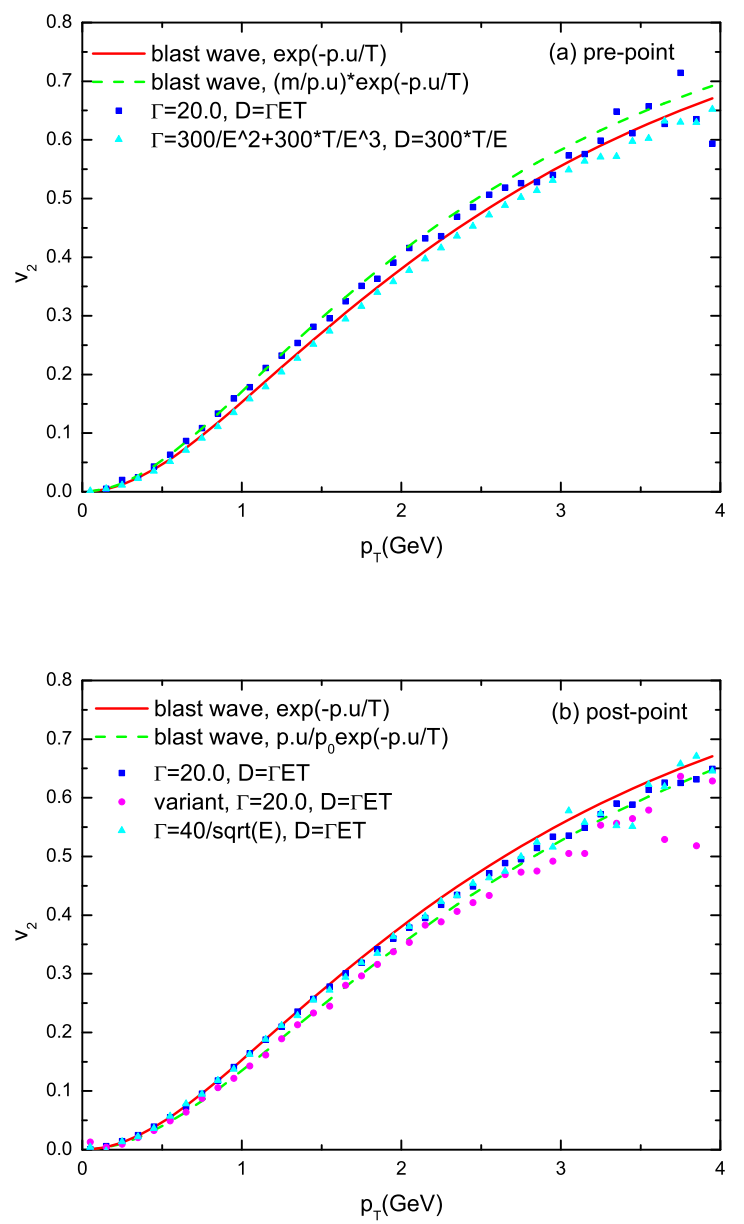

FIG. 4: Langevin simulation results for $v_{2}\left(p_{T}\right)$ for a heavy quark $(m=1.5 \mathrm{GeV})$ diffusing in a QGP $\left(T_{f}=0.18 \mathrm{GeV}\right)$ compared to direct blast-wave calculations with the flow field and temperature of the background medium (fireball). Upper panel: pre-point Langevin scheme; lower panel: post-point Langevin scheme.

\section{SUMMARY AND CONCLUSIONS}

In this work we have explored aspects of relativistic Langevin dynamics, in particular their uniqueness relative to a Fokker-Planck description and their manifestation in the presence of non-trivial medium-flow fields. Our perspective on these issues commenced from a microscopic theory for the interactions of a Brownian particle in a fluid of light particles, where the Fokker-Planck equation emerges from the Boltzmann equation with welldefined transport coefficients. We thus started by reestablishing the general constraint between the drag and diffusion coefficients in order for the asymptotic solution of the Fokker-Planck equation to converge to the Boltzmann-Jüttner distribution in the equilibrium limit. Based on this "master equation" we investigated two widely used Langevin realizations of the Fokker-Planck equation and explicitly obtained the equilibrium conditions for the coefficients in these schemes. It followed that both pre-point and post-point Langevin equations obey 
an equivalent Fokker-Planck equation in terms of the original drag and diffusion coefficients, thus illuminating the "Ito-Stratonovich dilemma", i.e., the pre-point and the post-point Langevin algorithm turn out to be equally adequate to describe the same micro-physics. We verified these results by explicit numerical simulations, recovering the Boltzmann-Jüttner distribution as the equilibrium limit of heavy test particles, if and only if the corresponding equilibrium condition is implemented exactly. We furthermore confirmed that the Langevin dynamics preserves the Lorentz invariance of the particles' phasespace distribution in the presence of collective-flow fields of the background medium: the particle's energy in the fluid rest frame, $E^{*}$, simply converts into $p \cdot u$, where $p$ is the particle's four momentum in the lab frame, and $u$ is the fluid four-velocity. This, in particular, showed the fulfillment of Lorentz-covariance through the two levels of approximation from full Boltzmann transport to the Langevin process. We believe that these insights are, in particular, useful for reliable simulations of heavy-quark diffusion in heavy-ion collisions, where the coupling of the Brownian particle to the rather subtle relativistic flow fields has become a quantitative tool to extract transport properties of the background medium.

\section{Acknowledgments}

This work has been supported by U.S. National Science Foundation under grants CAREER PHY-0847538, PHY0969394 and PHY-1306359, and by the A.-v.-Humboldt Foundation.
[1] S.R. de Groot, W. van Leeuwen, and C.G. van Weert, Relativistic Kinetic Theory: Principles and Applications (North-Holland, Amsterdam, 1980).

[2] J. Dunkel and P. Hänggi, Phys. Rev. E, 71, 016124 (2005); ibid, 72, 036106 (2005).

[3] J. Dunkel and P. Hänggi, Phys. Rep. 471, 1 (2009).

[4] F. Debbasch, K. Mallick, and J.P. Rivet, J. Stat. Phys. 88, 945 (1997).

[5] G. Chacon-Acosta and G.M. Kremer, Phys. Rev. E 76, 021201 (2007).

[6] B. Svetitsky, Phys. Rev. D 37, 2484 (1988).

[7] H. van Hees and R. Rapp, Phys. Rev. C 71, 034907 (2005).

[8] G. Moore and D. Teaney, Phys. Rev. C 71, 064904 (2005).

[9] M.G. Mustafa, Phys. Rev. C 72, 014905 (2005).

[10] P.B. Gossiaux, V. Guiho and J. Aichelin, J. Phys. G 31, S1079 (2005).

[11] R. Rapp and H. van Hees, in Quark Gluon Plasma 4, R. C. Hwa and X.-N. Wang (wds.), World Scientific, 111 (2010) arXiv:0903.1096 [hep-ph]].

[12] E.M. Lifshitz and L.P. Pitaevskii, Physical Kinetics (Pergamon Press, New York, 1981).

[13] N.G. van Kampen, Stochastic Processes in Physics and Chemistry (North-Holland, Amsterdam, 2003).
[14] D. Cubero, J. Casado-Pascual, J. Dunkel, P. Talkner, and P. Hänggi, Phys. Rev. Lett. 99, 170601 (2007).

[15] D.B. Walton and J. Rafelski, Phys. Rev. Lett. 84, 31 (2000).

[16] J.-Y. Ollitrault, Phys. Rev. D 46, 229 (1992).

[17] E.V. Shuryak, Prog. Part. Nucl. Phys. 62, 48 (2009).

[18] N.G. van Kampen, Physica 43, 244 (1969).

[19] F. Debbasch, J.P. Rivet and W.A. van Leeuwen, Physica A301, 181 (2001).

[20] F. Jüttner, Ann. Phys. (Leipzig) 34, 856 (1911).

[21] K. Ito, Mem. Am. Math. Soc. 4, 51 (1951).

[22] P. Arnold, Phys. Rev. E 61, 6091 (2000); ibid, 61, 6099 (2000).

[23] J. Dunkel, P. Hänggi and S. Weber, Phys. Rev. E 79, 010101 (2009).

[24] T. Koide and T. Kodama, Phys. Rev. E 83, 061111 (2011).

[25] A. Beraudo, A. De Pace, W.M. Alberico and A. Molinari, Nucl. Phys. A 831, 59 (2009).

[26] E. Braaten and M.H. Thoma, Phys. Rev. D 44, R2625 (1991).

[27] H. van Hees, V. Greco and R. Rapp, Phys. Rev. C 73, 034913 (2006).

[28] P.B. Gossiaux et al., arXiv:1102.1114v1 [hep-ph]. 